\title{
Overexpression of the dominant negative nbexo70d1 mutantion confers tolerance to salt stress in transgenic tobacco
}

\author{
N.N. TRINH ${ }^{*}$, H.T. LE ${ }^{2}$, and T.P. NGUYEN ${ }^{1}$ \\ Institute of Biotechnology and Food Technology, Industrial University of Ho Chi Minh City, Ho Chi Minh City, \\ Vietnam $^{l}$ \\ Institute for Environmental Science, Engineering and Management, Industrial University of Ho Chi Minh City, \\ Ho Chi Minh City, 700000, Vietnam ${ }^{2}$
}

\begin{abstract}
The vesicle trafficking process, which involves exocytotic and endocytotic pathways, has been reported to play a role in regulating plant responses to different environmental stresses. The Exo70 protein is important for the localization of the exocyst in the plasma membrane; however, its role in the physiology of stress tolerance is currently unclear. In this study, we characterized NbExo70D1, an Exo70 gene from tobacco (Nicotiana benthamiana). It was shown to have a role in the plant response to salt stress. More specifically, tolerance to salt stress is conferred by the overexpression of the dominant negative nbexo70d1 domain $D$ mutation in transgenic tobacco. In addition, a reduced accumulation of reactive oxygen species (ROS) under salt treatment was observed in the transgenic lines compared to the wild type. Treatment with diphenylene iodonium, an NADPH oxidase inhibitor, resulted in a decrease in salt stress-triggered ROS production in the roots of both wild type tobacco and transgenic tobacco. Furthermore, there was a reduction in NADPH oxidase activity in the transgenic plants under salt treatment, which indicates NbExo70D1 is involved in NADPH oxidase-mediated ROS production. We also characterized the tissue-specific expression patterns of NbExo70D1 during salt stress response by expressing the ProNbExo70D1- $\beta$-glucuronidase reporter construct in plants. Importantly, the GFP-NbExo70D1 fusion protein was localized in both the plasma membrane and the cytoplasm; expressing the dominant negative mutation disrupted the interaction between NbExo70D1 protein and the plasma membrane. Overall, our study suggests that Exo70 plays an important role in regulating the production and transmission of ROS as part of a salt stress response in plants.
\end{abstract}

Additional key words: diphenylene iodonium, $\beta$-glucuronidase, NADPH oxidase, Nicotiana benthamiana, reactive oxygen species.

\section{Introduction}

Vesicular trafficking plays a crucial role in protein localization and movement, signal transduction, and it is involved in many developmental processes (Yao and Xue 2011). Vesicular trafficking is mediated by distinct exocytic and endocytic routes in plant cells. As an essential function for plant growth, exocytosis involves the fusion of the Golgi derived vesicles with the target plasma membrane to release the vesicle content into the extracellular space (Li et al. 2010). Vesicle tethering is an important targeting step that is mediated either by long tethering proteins or by a multi-subunit tethering complex (Cai et al. 2007). The exocytotic vesicle tethering involves the exocyst octametic protein $(\operatorname{Sec} 3 p, \operatorname{Sec} 5 p$, Sec6p, Sec8p, Sec10p, Sec15p, Exo70p, and Exo84p). This protein complex was originally discovered in yeast and animal cells (TerBush et al. 1996, Hsu et al. 2004, Cai et al. 2007, Wu et al. 2008). The C-terminal domain $\mathrm{D}$ of the Exo70 subunit of the exocyst complex directly interacts with phosphatidylinositol 4,5-biphosphate [PtdIns(4,5)P2] via their positively charged amino acids,

Submitted 13 March 2018, last revision 25 February 2019, accepted 5 March 2019.

Abbreviations: BSA - bovine serum albumin; CM-H ${ }_{2}$ DCFDA - 5-(and-6)-chloromethyl-2',7'-dichlorodihydrofluorescein diacetate acetyl ester; DAB - 3,3'-diaminobenzidine; DPI - diphenylene iodonium; ER - endoplasmic reticulum; FM4-64 - styryl dye N-(3triethylammoniumpropyl)-4-(6-(4-(diethylamino) phenyl)hexatrienyl)pyridinium dibromide; GFP - green fluorescent protein; GUS $\beta$-glucuronidase; MS - Murashige nd Skoog; MV - methyl viologen; NBT - nitroblue tetrazolium; PtdIns(4,5)P2 - phosphatidylinositol4,5-biphosphate; PVDF - polyvinylidene difluoride; ROS - reactive oxygen species; SDS-PAGE - sodium dodecylsulfate-polyacrylamide gel electrophoreses; XTT - sodium,3'-[1-[phenylamino-carbonyl]-3,4-tetrazolium]-bis(4- methoxy-6-nitro)benzenesulfonicacid hydrate.

Acknowledgments: This work was supported by research grants from the Industrial University of Ho Chi Minh City, Vietnam in 2016 (IUH.VSH16/17). We thank Prof. Claire Remacle (University of Liége, Belgium) for her careful review of the manuscript and English editing. We are grateful to Prof. Huang Hao Jen (National Cheng Kung University, Tainan, Taiwan) for his support.

* Corresponding author; e-mail: trinhngocnam@iuh.edu.vn 
which are evolutionarily conserved across various species, to anchor the exocyst to the plasma membrane (Dong et al. 2005, He et al. 2007, Liu et al. 2007).

Hála et al. (2008) described the angiosperm exocyst complex and confirmed the presence of homologs for all eight subunits of the complex. The exocyst complex component Exo70 is coded by a single gene in yeast and in most animals. In contrast, plants have evolved numerous Exo70 genes in their genomes with unclear functions (Li et al. 2010). Nicotiana benthamiana, Solanum lycopersicum, Arabidopsis thaliana, and Oryza sativa have 44, 22, 23, and 47 Exo70 genes, respectively (Chong et al. 2010, Cvrckova et al. 2012, Rawat et al. 2017, Du et al. 2018). The cellular role of the exocyst has been extensively studied in yeast and animals (Hsu et al. 2004). The exocyst is involved when physiological or environmental circumstances activate extensive exocytosis to support a rapid polarized growth. This includes a rapid reproduction via budding in Saccharomyces cerevisiae (TerBush and Novick 1995), hyphal tip growth in Candida albicans (Li et al. 2007), the outgrowth of cultured neurites (Hazuka et al. 1999, Pommereit and Wouters 2007), and membrane trafficking to the leading growing edge of migrating mammalian epithelial cells (Rossé et al. 2006, Zuo et al. 2006). Study on the role of the exocyst complex proteins in plant growth, however, had only recently begun.

There are only few studies regarding the role of exocytosis in the response to abiotic stress in plants (Mazel et al. 2004, Zhao et al. 2018). In both Arabidopsis and barley, metal tolerance involves exocytosis via metalcontaining vesicles (Hirschi et al. 2000). Deoxyribonucleic acid microarray expression analyses revealed that AtExo 70 gene expressions generally increase following pathogen infections, hormone treatments, and abiotic stress. There may also potentially be other roles involving the exocyst in these processes (Chong et al. 2010). Li et al. (2010) found that several of Exo70 genes in the A. thaliana (AtExo70A2, AtExo70B2, At Exo70H1, and AtExo70H4) have an increased expression in response to abiotic stress treatments. Genome wide association study revealed that salinity tolerance is associated with one of the Exo70 genes (At5g52340) in A. thaliana (Julkowska et al. 2016). AtExo70B1 and AtExo70B2 are positive regulators of stomatal movement in drought response (Hong et al. 2016, Seo et al. 2016). In addition, AtEx70B1 and AtExo70E2 were found to be involved in autophagy related membrane trafficking as part of the stress response in plants (Kulich et al. 2013, Lin et al. 2015). AtExo70B1 is internalized into the central vacuole and co-localize with autophagosomal marker ATG8f, which is an autophagy protein involved in a system that is similar to the ubiquitin system. Other study indicated that Exo7 can also play a regulatory function not related to membrane trafficking (Sekereš et al. 2017). In our earlier work on rice using transcriptomic analysis, we found that genes involved in vesicle trafficking, such as OsExo 70 s, are regulated specifically by heavy metal stress (Lin et al. 2013).

The generation of reactive oxygen species (ROS) is one of the major plant responses to abiotic stresses, including salt stress. The main intracellular sources of ROS in plants are the endoplasmic reticulum (ER), peroxisomes, mitochondria, chloroplasts, and plasma membrane (PérezPérez et al. 2012). Reactive oxygen species generated by plasma membrane NADPH oxidase play crucial roles in plant response to salt stress (Mazel et al. 2004, Leshem et al. 2006, 2007). The NADPH oxidase receives electrons from NADPH at the cytosolic side of the membrane. It then donates them to molecular oxygen $\left(\mathrm{O}_{2}\right)$ at the other side of the membrane, thus producing superoxide anions outside of the plasma membrane (Sato et al. 2001). Upon formation, the superoxide anions spontaneously or enzymatically dismutates to $\mathrm{H}_{2} \mathrm{O}_{2}$. Previous studies reported that vesicle trafficking is involved in ROS production by NADPH oxidase in response to environmental stresses in plants (Mazel et al. 2004, Leshem et al. 2007, Wong et al. 2007).

In our previous works, we found that the vesicle trafficking-related gene NbExo70D1 (GenBank: KF280306.1, gene ID: NbS00034553g0002; Du et al. 2018) was involved in ROS production under heavy metal stresses (Trinh et al. 2014). Here, we present a function study of the vesicle trafficking-related (NbExo70D1) gene in tolerance to salt stress. With this aim, we conducted an overexpression analysis of the dominant negative nbexo70d1 domain $D$ mutantion in transgenic tobacco plants. We tried to determine if mutation of positively charged residues at the C-terminus of NbExo70D1 results in the disruption of membrane targeting, which subsequently decreases ROS production by NADPH oxidase and increase tolerance to salt stress.

\section{Materials and methods}

Plants, growth conditions, and treatments: Tobacco (Nicotiana benthamiana Domin) was used for stable and transient transformation using the Agrobacterium tumefaciens strain GV3101. For root growth measurements, 5-d-old seedlings cultured on a one-half strength solid Murashige and Skoog (MS) medium (Duchefa Biochemie, Haarlem, Netherlands) were transferred to the onehalf strength MS medium supplemented with different concentrations of $\mathrm{NaCl}(0,100,200$, and $300 \mathrm{mM}), \mathrm{KCl}(0$, $100,200$ and $300 \mathrm{mM})$, and sorbitol (0, 200, and $400 \mathrm{mM})$. The tobacco seedlings were cultured in a growth chamber at a temperature of $25 \pm 2{ }^{\circ} \mathrm{C}$, cool white fluorescent lamps (an irradiance of $100 \mu \mathrm{mol} \mathrm{m} \mathrm{m}^{-2} \mathrm{~s}^{-1}$ ) and a 16-h photoperiod. Root length was recorded after $5 \mathrm{~d}$ of treatment. There were three biological replicates in each experiment.

For the methyl viologen (MV) treatment, leaves of 3 -week-old $N$. benthamiana seedlings were soaked in the MS medium solution containing $10 \mu \mathrm{M} \mathrm{MV}$, incubated at $25^{\circ} \mathrm{C}$ for $2 \mathrm{~d}$, and then put in a growth chamber.

Expression constructs: The $1.5 \mathrm{~kb}$ promoter region upstream from the start codon of the NbExo70D1 gene was amplified using $N$. benthamiana genomic DNA as a template. The NbExo70D1 promoter fragment was cloned into the pENTR-D TOPO (Invitrogen, Carlsbad, CA, USA) and left/right att sites subcloned into the plant destination vector pHGWFS7 (Plant Systems Biology, Ghent, Belgium) 
to generate the ProNbExo70D1- $\beta$-glucuronidase (GUS) reporter construct. The transgenic lines were confirmed by PCR using promoter-specific primers.

The overlapping-extension PCR method (Ho et al. 1989, An et al. 2005) was carried out to generate specific point mutations in NbExo70D1 domain D (Table 1Suppl.). Corresponding primers are listed in Table 1 Suppl. Two separate PCR reactions were performed using $p f u$ polymerase (Promega, Madison, WI, USA) and primer pairs using $\mathrm{NbExo} 70 \mathrm{D} 1 \mathrm{cDNA}$ as a template. For the fusion of the two sequences, the sequences were gel purified and then mixed in equal proportions in a PCR reaction without primers. A PCR-mediated assembly was performed using pfu polymerase mixed with $1 \times$ reaction buffer consisting of $20 \mathrm{mM}$ Tris- $\mathrm{HCl}, 10 \mathrm{mM} \mathrm{KCl}, 10 \mathrm{mM}\left(\mathrm{NH}_{4}\right)_{2} \mathrm{SO}_{4}$, $2 \mathrm{mM} \mathrm{MgSO}_{4}, 0.1 \mathrm{mg} \mathrm{cm}^{-3}$ nuclease-free BSA, $0.1 \%$ (v/v) Triton ${ }^{\circledR} \mathrm{X}-100,0.2 \mathrm{mM}$ dNTPs, and templates. The fusion was run under the following conditions: at $95^{\circ} \mathrm{C}$ for $2 \mathrm{~min}$ followed by 15 cycles at $95^{\circ} \mathrm{C}$ for $1 \mathrm{~min}, 55^{\circ} \mathrm{C}$ for $1 \mathrm{~min}$, and $72{ }^{\circ} \mathrm{C}$ for $5 \mathrm{~min}$, with a final polymerization at $72{ }^{\circ} \mathrm{C}$ for $10 \mathrm{~min}$. The PCR amplifications were performed using pfu polymerase mixed with the $1 \times$ reaction buffer, $0.1 \mathrm{mM}$ dNTPs, $0.2 \mu \mathrm{M}$ each of outer primers, and templates. The PCR amplification was run under the following conditions: at $95{ }^{\circ} \mathrm{C}$ for $2 \mathrm{~min}$ followed by 30 cycles at $95{ }^{\circ} \mathrm{C}$ for $1 \mathrm{~min}, 55^{\circ} \mathrm{C}$ for $1 \mathrm{~min}$, and extension at $72{ }^{\circ} \mathrm{C}$ for $5 \mathrm{~min}$, followed by a final polymerization at $72{ }^{\circ} \mathrm{C}$ for $10 \mathrm{~min}$. The resulting full-length mutant fragments were cloned into the pENTR-D TOPO and subcloned into the plant destination vector $\mathrm{pH} 7 \mathrm{WG} 2$ by left/right att site recombination reaction (Invitrogen, Carlsbad, CA, USA). The orientation and integrity of the constructs was confirmed by sequence analysis.

A PCR was used to introduce restriction sites into a complete open reading frame NbExo70D1 cDNA (Genbank accession number: KF280306) to facilitate directional cloning into the pGDG vector (Goodin et al. 2002). The HindIII and BamHI sites were used to generate the NbExo70D1:green fluorescence protein $(G F P)$ in-frame fusion construct under the control of the Cauliflower mosaic virus 35S (CaMV 35S) promoter. The NbExo70D1-RE full-length sequence primer set was used for this amplification (Table 1 Suppl.). In this construct, the GFP tag was positioned at the N-terminal of NbExo70D1. Nbexo70d1 domain $D$ mutation was cloned into the pENTR-D TOPO vector and subcloned into the plant destination vector pSITEII-4C1 (Martin et al. 2009) by left/right att site recombination reaction recombination reaction to generate Venus fusion proteins.

NbATG $8 f$ (KU561372.1) was amplified by PCR using $p f u$ polymerase under the following conditions: predenaturation at $95{ }^{\circ} \mathrm{C}$ for $2 \mathrm{~min}$ followed by 30 cycles of denaturation at $95{ }^{\circ} \mathrm{C}$ for $1 \mathrm{~min}$, annealing at $50{ }^{\circ} \mathrm{C}$ for $1 \mathrm{~min}$, extension at $72{ }^{\circ} \mathrm{C}$ for $1 \mathrm{~min}$, and a final polymerization at $72{ }^{\circ} \mathrm{C}$ for $10 \mathrm{~min}$. The resulting fulllength NbATG $8 f$ product was cloned into the pENTR-D TOPO vector and subcloned into the pSITE-4CA vector.

Transient expression of fluorescent proteins in tobacco leaves: Each expression vector harboring the
GFP:NbExo70D1-, YFP:nbexo70d1 domain D mutation-, or RFP:NbATG8-derived construct was introduced into the Agrobacterium strain GV3101 by electroporation as described Bhaskar et al. (2009). The cell suspensions were inoculated into the leaves of 2- to 4-week-old $N$. benthamiana plants on the abaxial epidermal surface. Fluorescence of transformed leaves was observed at $72 \mathrm{~h}$ post inoculation by confocal laser-scanning microscopy (LSM780, Carl Zeiss, Jena, Germany). Fluorescence in the plasma membrane and the intracellular membrane of the abaxial epidermal cells was detected using a styryl probe dye N-(3-triethylammoniumpropyl)-4-(6-(4(diethylamino) phenyl)hexatrienyl) pyridinium dibromide (FM4-64) (Molecular Probes, Invitrogen, Carlsbad, CA, USA). The dye was loaded at $10 \mu \mathrm{M}$ concentration, left for $60 \mathrm{~min}$, and then washed twice in the same buffer.

For quantification of NbExo70D1 localization in the membrane, NbExo70D1 from each section was examined, and the dots were counted three times. The number of NbExo70 dots counted was then averaged. Plasma membrane and cytoplasmic localization was presented as the percentage of NbExo70D1. We used three independently transformed lines for each genotype.

Plant transformation: The nbex70d1 domain $D$ mutation and the ProExo70D1-GUS reporter constructs were inserted into the A. tumefaciens strain GV 3101. Transformation of $N$. benthamiana was carried out using the leaf disc method as described previously in Trinh et al. (2017). Transgenic plants regenerated from transformed calli were transferred to Vriezenveen $70 \mathrm{~L}$ substrates (Potgrond Vriezenveen, Westerhaar, The Netherlands) and grown in a growth chamber at $27^{\circ} \mathrm{C}$ and other conditions as mentioned above. The seeds of 20 independent lines were harvested from these primary transformants.

Isolation of RNA and real-time semi-quantitative PCR analysis: Total RNA was extracted from the leaf tissues of 3-week-old $N$. benthamiana plants with an RNeasy plant mini kit (Qiagen, Hilden, Germany) and treated with DNase I (Qiagen) to eliminate genomic DNA contamination. It was then purified and concentrated by using a RNeasy MinElute Cleanup kit (Qiagen). The yield and purity of total RNA were detected by a spectrophotometer (NanodropND 2000, Nanodrop Technologies, Wilmington, DE, USA). The RNA samples had a concentration of $2 \mathrm{mg} \mathrm{cm}^{-3}$ and absorbance ratios $A_{260 / 280}$ and $A_{260 / 230}>2$. These were used for further analyses.

To determine the expression of NbExo70D1, NbrbohA, and NbrbohB genes under salt stress, 7-d-old tobacco seedlings were treated with $200 \mathrm{mM} \mathrm{NaCl}$ at six different time points. Semi-quantitative PCR analysis was performed using a specific primer set (NbExo70D1 partial sequence primers listed in Table 1 Suppl.). The NbrbohA and $N b r b o h B$ primers were used to determine the level of gene expressions for Nbrboh under salt stress [described in Yoshioka et al. (2003)]. In $N$. benthamiana, the Elongation factor 1- $\alpha(N b E F 1-\alpha)$ gene was used as an internal loading control to determine the efficiency of the semi quantitative 
PCR among different samples. Three biological and three technical replicates of each sample were used for analysis.

The expressions of the combined NbExo70D1 and nbexo70d1 domain $D$ mutation within wild type or dominant negative nbex70d1 domain $D$ mutant plants, respectively, were identified using semiquantitative PCR using primers listed in Table 1 Suppl. The forward primer 5'-CAGAGATCAACCTGGGTGAG-3' and the reverse primer 5'-CCACAAAACAATTCAAAGAATGTCC-3' were derived for the 3 '-untranslated region of the NbExo70D1 mRNA. These were used to analyze the expression of the endogenous $N b E x o 70 D 1$ mRNA in the transgenic plants. The expression of the NbEF1- $\alpha$ gene was used as an internal control. There were three biological replicates that were grown in the same growth chamber to minimize experimental and sample-to-sample variation.

Immunological detection of proteins: Tobacco leaf tissues were separately ground to a fine powder using a mortar and pestle. The powder was homogenized in a buffer containing $1 \mathrm{M}$ sucrose, $10 \mathrm{mM}$ HEPES buffer ( $\mathrm{pH}$ 7.2), $5 \mathrm{mM} \mathrm{MgCl}_{2}, 1 \mathrm{mM} \mathrm{Na} \mathrm{NaDTA}_{2}$, $10 \mathrm{mM}$ 2-mercaptoethanol, $0.1 \mathrm{mM}$ phenyl-methylsulphonyl fluoride (Sigma, St. Louis, MO, USA) and $1 \mathrm{mM}$ benzamidine (Sigma). The homogenates were centrifuged at $13400 \mathrm{~g}$ and $4{ }^{\circ} \mathrm{C}$ for $1 \mathrm{~h}$. Then the protein concentration of the supernatant was determined using the Bio-Rad Dc protein assay (Bio-Rad, Hercules, CA, USA) at an absorbance of $750 \mathrm{~nm}$. Fifteen micrograms of protein from each sample was separated by $12 \%(\mathrm{~m} / \mathrm{v})$ sodium dodecylsulfate-polyacrylamide gel electrophoresis run at $80 \mathrm{~V}$ using a mini-PROTEAN II system (Bio-Rad). Equal loading was ensured using Coomassie brilliant blue staining the gels. The proteins were electro-transferred onto polyvinylidene difluoride membranes (PVDF, Millipore, Billerica, MA, USA) using a Bio-Rad system. The membranes were used in immunoblotting. Briefly, the blots were first blocked with $5 \%(\mathrm{~m} / \mathrm{v})$ non-fat dried milk in phosphate buffered saline at $4{ }^{\circ} \mathrm{C}$ overnight, and then were washed twice with PBST (phosphate-buffered saline with $0.05 \%(\mathrm{v} / \mathrm{v})$ Tween-20). The membranes were incubated with GFP anti-serum in PBST and $1 \%(\mathrm{~m} / \mathrm{v})$ bovine serum albumin for $1 \mathrm{~h}$ at room temperature. The rabbit anti-GFP (N-terminal) peptide antibody (\#G1544, Sigma-Aldrich, Munich, Germany) was dissolved to a final concentration of 1:5000. After washing the membranes with PBST, the immune reactive proteins were detected by incubation with horse radish peroxidase-conjugated antirabbit IgG (Promega) at a final dilution of 1:5000. Western blot analyses were performed using an ECL detection system (RPN 2106; Amersham Pharmacia Biotech, Piscataway, NJ, USA).

Histochemical detection of GUS activity: Histochemical GUS analysis in transgenic $N$. benthamiana plants carrying the reporter constructs was firstly examined in 20 independent T1 plants to identify lines with a consistent expression pattern; a procedure outlined in Jefferson et al. (1987). Detailed analyses were performed in the T2 generation. Briefly, plant tissues were incubated in the dark at $37{ }^{\circ} \mathrm{C}$ for $16 \mathrm{~h}$ in $0.1 \mathrm{M} \mathrm{NaPO}_{4}$ buffer $(\mathrm{pH} 7.0)$ containing $5 \%(\mathrm{v} / \mathrm{v})$ methanol, $0.01 \%(\mathrm{v} / \mathrm{v})$ Triton $X-100$, $0.5 \mathrm{mM} \mathrm{K}_{3}\left(\mathrm{Fe}(\mathrm{CN})_{6}\right), 0.5 \mathrm{mM} \mathrm{K}_{4}\left(\mathrm{Fe}(\mathrm{CN})_{6}\right)$, and $1 \mathrm{mg} \mathrm{cm}^{-3}$ 5-bromo-4-chloro-3-indolyl glucuronide. After incubation, the plant tissues were washed after GUS staining using $95 \%(\mathrm{v} / \mathrm{v})$ ethanol and glacial acetic acid (3:1, v/v) to remove chlorophyll before examination under a Leica MZ12.5 stereomicroscope (Leica, Wetzlar, Germany).

In situ detection of ROS: The tobacco seedlings were taken out from a half-strength MS agar medium after $5 \mathrm{~d}$ and transferred to the MS medium with or without the addition of $200 \mathrm{mM} \mathrm{NaCl}$. After $24 \mathrm{~h}$, the generation of ROS was detected using a Leica MPS60 fluorescent microscope with a GFP filter (excitation at 450 to $490 \mathrm{~nm}$, emission at 500 to $530 \mathrm{~nm}$ ) after the addition of $10 \mu \mathrm{M}$ 5-(and-6)-chlormethyl-2',7'-dichlordihydrofluorescein diacetate acetyl ester (CM- $\mathrm{H}_{2} \mathrm{DCF}-\mathrm{DA}$, Molecular Probes, Invitrogen, Carlsbad, CA, USA) for $30 \mathrm{~min}$. Fluorescence and bright-field images were taken with a CoolSNAP Cooled CCD camera (CoolSNAP 5.0, North Reading, MA, USA).

Superoxide anion and $\mathrm{H}_{2} \mathrm{O}_{2}$ content in the $N$. benthamiana seedlings and in leaves (grown on the onehalf strength MS medium with or without $\mathrm{NaCl}$ ), were visually detected by nitro-blue tetrazolium (NBT; Sigma, St. Louis, MI, USA) and 3,3'-diaminobenzidine (DAB; Sigma), respectively (Thordal-Christensen et al. 1997). To detect superoxide anion radicals, $0.5 \mathrm{mg} \mathrm{cm}^{-3} \mathrm{NBT}$ was supplied with $10 \mathrm{mM} \mathrm{NaN}_{3}$ in $10 \mathrm{mM}$ potassium phosphate buffer ( $\mathrm{pH}$ 7.8). For $\mathrm{H}_{2} \mathrm{O}_{2}$ staining, the seedlings were placed in $1 \mathrm{mg} \mathrm{cm}^{-3} \mathrm{DAB}$ solution with $10 \mathrm{mM}$ MES buffer ( $\mathrm{pH} 3.8$ ) for $8 \mathrm{~h}$ in darkness.

NADPH oxidase activity assays: Activity of NADPH oxidase was measured by sodium,3'-[1-[phenylaminocarbonyl]-3,4-tetrazolium]-bis(4- methoxy-6-nitro) benzenesulfonicacid hydrate (XTT) assay and by ingel assay. Tobacco seedlings (7-d-old) were pre-treated with or without $100 \mu \mathrm{M}$ diphenylene iodonium (DPI), an NADPH oxidase inhibitor. After $1 \mathrm{~h}$, they were treated with $200 \mathrm{mM} \mathrm{NaCl}$ for $30 \mathrm{~min}$. The seedlings ( $1 \mathrm{~g}$ ) were ground in liquid nitrogen using a mortar and pestle and then mixed with $0.05 \mathrm{M}$ potassium phosphate buffer (pH 5.8) to extract NADPH oxidase. The homogenized tissue was centrifuged at $13400 \mathrm{~g}$ and $4{ }^{\circ} \mathrm{C}$ for $20 \mathrm{~min}$. The supernatant was assayed for NADPH oxidase activity. An XTT assay mixture $\left(1 \mathrm{~cm}^{3}\right)$ consisted of $50 \mathrm{mM}$ Tris-HCl buffer (pH 7.5), $0.5 \mathrm{mM} \mathrm{XTT} \mathrm{(Sigma),} \mathrm{and}$ $0.1 \mathrm{mM}$ NADPH (Sigma). An NADPH addition initiated a reaction, and XTT reduction was determined at $470 \mathrm{~nm}$ (Sagi and Fluhr 2001). The in-gel assay used to assess NADPH oxidase activity was performed according to the procedures described in Sagi and Fluhr (2001).

Leaf disc salt stress assay: Leaf discs of $9 \mathrm{~mm}$ in diameter were excised from fully expanded leaves of transgenic plants with nbexo70d1 domain $D$ mutation and from wild type plants of the same age. The leaf discs were floated on $2 \mathrm{~cm}^{3}$ of a liquid MS medium supplemented with various 


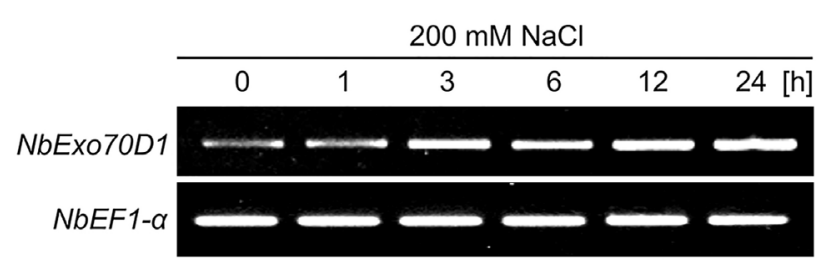

Fig. 1.Expression patternof NbExo70D1 inNicotianabenthamiana seedlings after treatment with $200 \mathrm{mM} \mathrm{NaCl}$ for 0 to $24 \mathrm{~h}$. NbEF $1-\alpha$ was used as an internal loading control. The experiments were repeated three times in independent samples and similar results were obtained.

concentrations of $\mathrm{NaCl}(0,100,200$, and $300 \mathrm{mM})$ for $4 \mathrm{~d}$ in a growth chamber. There were three replicate leaf discs for each concentration. The growth chamber was set at $27{ }^{\circ} \mathrm{C}$, a $70 \%$ air humidity, a 16-h photoperiod, and an irradiance of $100 \mu \mathrm{mol} \mathrm{m} \mathrm{m}^{-2} \mathrm{~s}^{-1}$. The effect of salt stress on the leaf discs was evaluated by quantifying chlorophyll content from nine leaf discs according to the procedure described in Arnon (1949).

\section{Results and discussion}

To analyze the expression profile of NbExo70D1 semi quantitative PCR was performed in the tobacco seedlings.
The transcription of NbExo70D1 was induced within $1 \mathrm{~h}$ under $\mathrm{NaCl}$ treatment (Fig. 1) and continuously increased and peaked at $24 \mathrm{~h}$ after exposure. This result suggests that NbExo70D1 may be involved in the response to salt stress.

Using transgenic plants with the GUS reporter constructs, Li et al. (2010) found that the AtExo70 genes are primarily expressed in cells that are potentially exocytosisactive such as cells at growing tips, elongating cells, guard cells, and developing xylem elements. In contrast, no expression is observed in the cells of mature organs such as well-developed leaves, stems, sepals, and petals. In the plant response to heavy metal stress, we previously demonstrated that the NbExo70D1 gene is strongly induced with $\mathrm{Cu}$ (II) treatment in seedlings and leaves of $N$. benthamiana (Lin et al. 2013). In order to determine whether the different roles of NbExo70D1 in salt stress response corresponds to differential expression patterns, the GUS reporter gene fusion (ProNbExo70D1::GUS) was constructed using approximately $1.5 \mathrm{~kb}$ of the upstream regulatory region for the NbExo70D1 gene. The microscopic studies show a strong GUS expression in primary roots, lateral roots, shoot apical meristems, cotyledons, and along vascular bundles of transgenic seedlings under salt stress (Fig. 2A). $\beta$-Glucuronidase staining was also observed in petals, sepals, ovary, stigma, anthers, and leaves of the transformed plants under salt stress (Fig. 2B). Expression of GUS was not observed
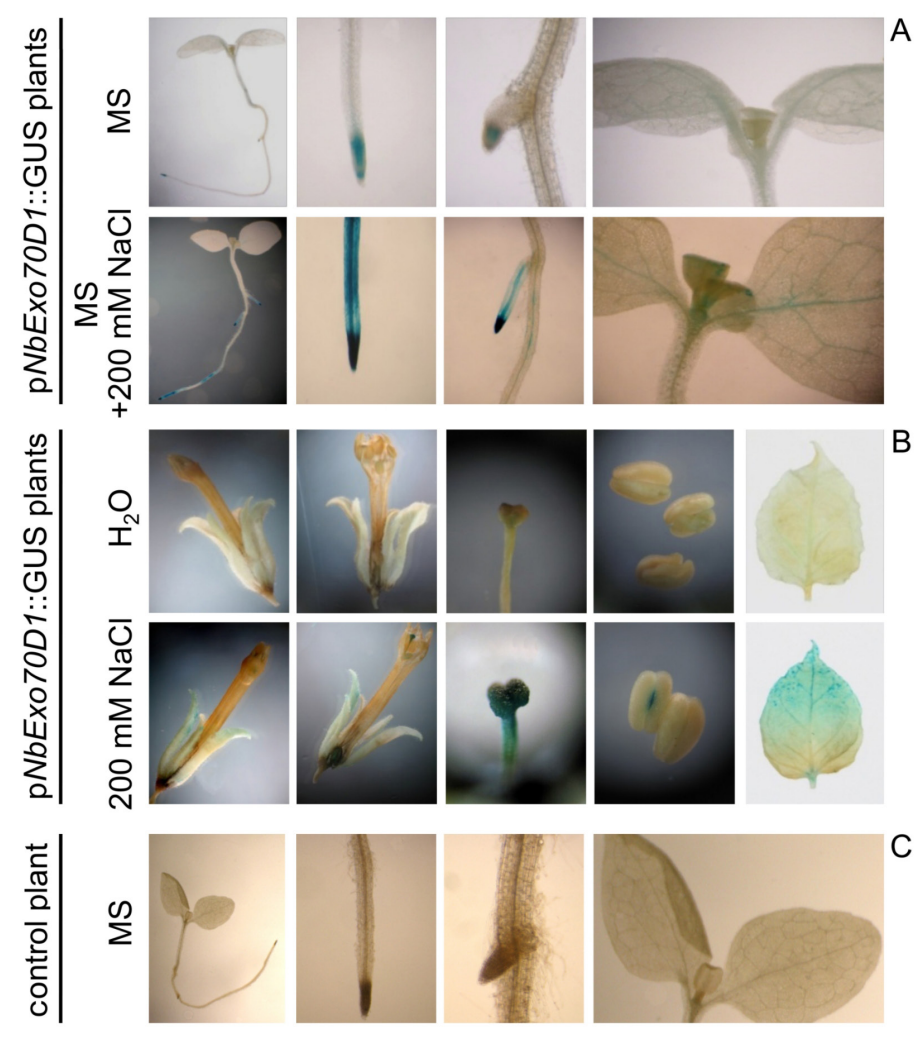

Fig. 2. Tissue-specific expression patterns in Nicotiana benthamiana plants transformed with an Exo70 promoter driven by the $\beta$-glucuronidase (GUS) reporter gene (a pNbExo 70D1::GUS construct) in response to salt stress. $A$ - GUS expression patterns in 5-d-old transgenic seedlings treated with 0 or $200 \mathrm{mM} \mathrm{NaCl}$ for $24 \mathrm{~h}$. $B$ - GUS expression patterns in reproductive organs and leaves of transgenic plants treated with 0 or $200 \mathrm{mM} \mathrm{NaCl}$ for $24 \mathrm{~h}$. $C$ - GUS activity was absent in 5-d-old wild-type seedlings. In all cases, there were at least 10 replicate samples, and similar results were obtained. 


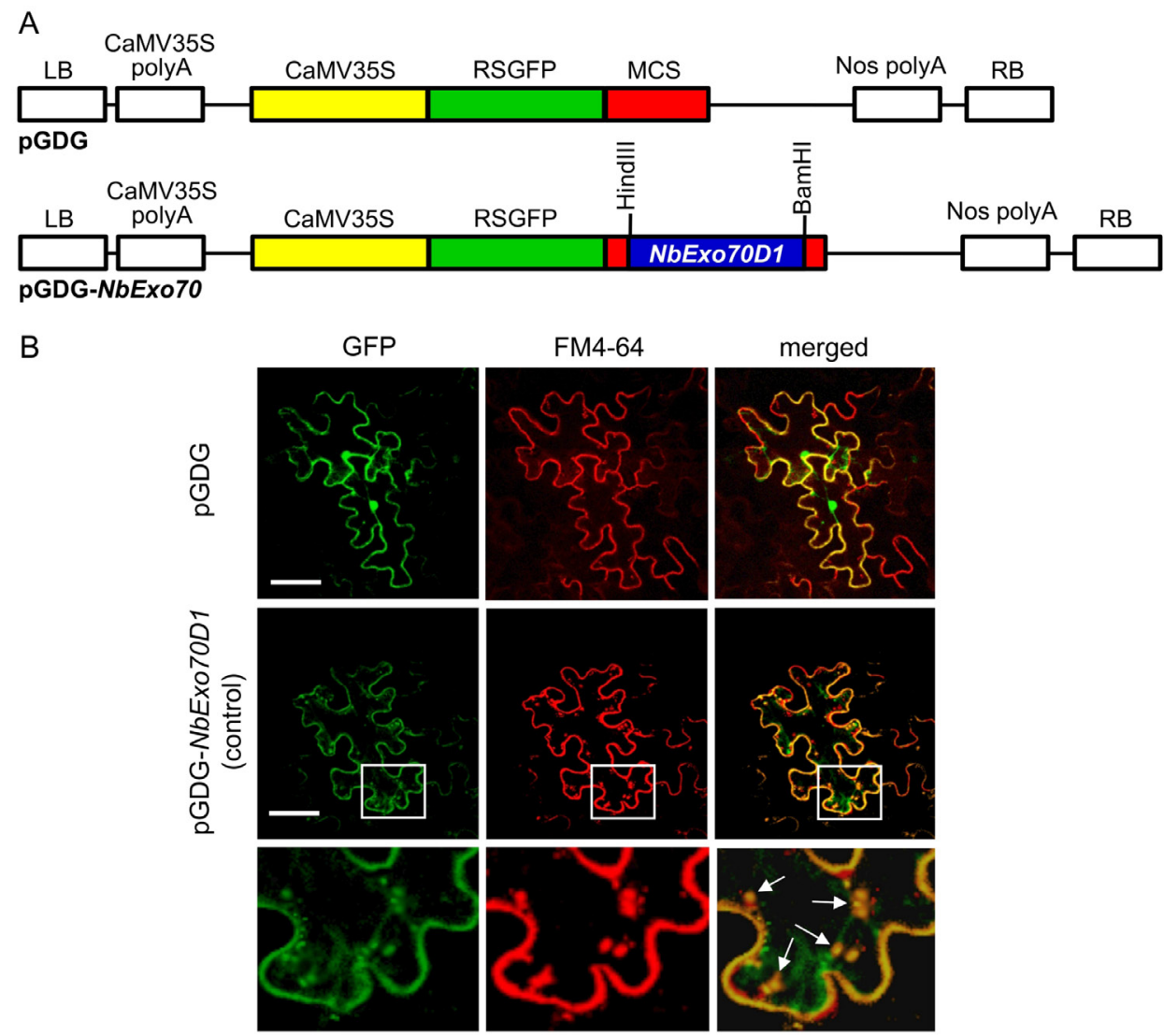

Fig. 3. The subcellular localization of NbExo70D1 in Nicotiana benthamiana leaf epidermal cells. GFP-NbExo70D1 was transiently expressed in epidermal cells of $N$. benthamiana leaves after agroinfiltration. $A$ - A schematic diagram of the constructs used for transformation of $N$. benthamiana leaf epidermal cells. $B$ - Confocal laser-scanning microscopy images of tobacco leaf epidermal cells expressing $p G D G$ and the typical intracellular localization of NbExo70D1. A higher magnification of the confocal image is shown in the squares. Plasma membranes and intracellular membranes were stained by the FM4-64 dye. Arrows indicate the secretory vesicle labeled by FM4-64. White bars $-10 \mu \mathrm{m}$.

in the tissues of non-transgenic control plants (Fig. 2C). These results indicate that NbExo70D1 has differential roles in salt stress responses in different tissues.

To evaluate the evolutionary relationship of NbExo70D1 within the Exo70 protein family, a phylogenetic tree was constructed using the MEGA 7.0 software based on the amino acid sequences from various Exo70 members from $A$. thaliana. NbExo70D1 clustered with the group D AtExo70 protein and was closely related to AtExo70D1 (Fig. 1 Suppl.). Furthermore, we generated sequence alignments of NbExo70D1 with Exo70s from other organisms (Fig. 2 Suppl.). The NbExo70D1 protein sequence is composed of four domains (A, B, C, and D). Domain $\mathrm{D}$ is the most evolutionarily conserved region in Exo70 (Dong et al. 2005, He et al. 2007). It contains a number of basic residues that cluster into an electropositive patch on the surface of the C-terminus. Exo70 binds to phospholipids through the positively charged surface patch on domain $\mathrm{D}$ at the $\mathrm{C}$-terminus (He et al. 2007). In a previous study where we used virus induced-gene silencing, we found that silencing NbExo70D1 may reduce ROS production and increase tolerance to heavy metal stress in plants (Trinh et al. 2014). In addition, the $e F P$ database showed that $A$. thaliana Exo70D1, located at the same branch point with $\mathrm{NbExo} 70 \mathrm{D} 1$, is induced after 6-h exposure to $150 \mathrm{mM}$ of $\mathrm{NaCl}$ (Kilian et al. 2007). To investigate whether vesicle tethering by NbExo70D1 at the plasma membrane plays an important role in plant response to salt stress, we generated nbexo70d1 domain $D-1$ mutations at the Arp $2 / 3$ complex binding site, and the nbexo70d1 domain D-2 mutations at the PtdIns $(4,5)$ $\mathrm{P} 2$ binding site of NbExo70D1 domain $\mathrm{D}$ by site-directed mutagenesis. In the mutants, three positively charged amino acid residues on NbExo70D1 domain $\mathrm{D}$ were replaced by non-polar amino acid residues (i.e., alanine; Fig. 3 Suppl.). Twenty transgenic plants of each mutant were produced. Three transgenic lines from each mutant were chosen for further analyses. Semi-quantitative PCR showed that all nbexo70d1 domain $D$ mutation expressing plants had a higher amount of mutant mRNA in comparison with the wild type NbExo70D1 (Fig. 4 Suppl.). The expression of the NbExo70D1 gene remained unaltered in these lines. Surprisingly, all of the nbexo70d1 domain $D$ mutation plants did not differ from the wild type plants in the overall morphology and development throughout their life cycle (Fig. 4 Suppl.).

To evaluate the possible effects of the nbexo70d1 domain $D$ mutation on salt tolerance, the wild type and 


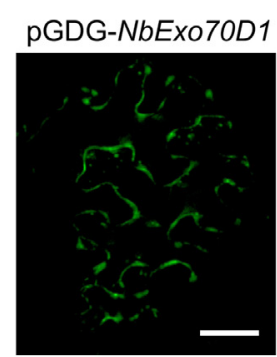

pRFP-ER
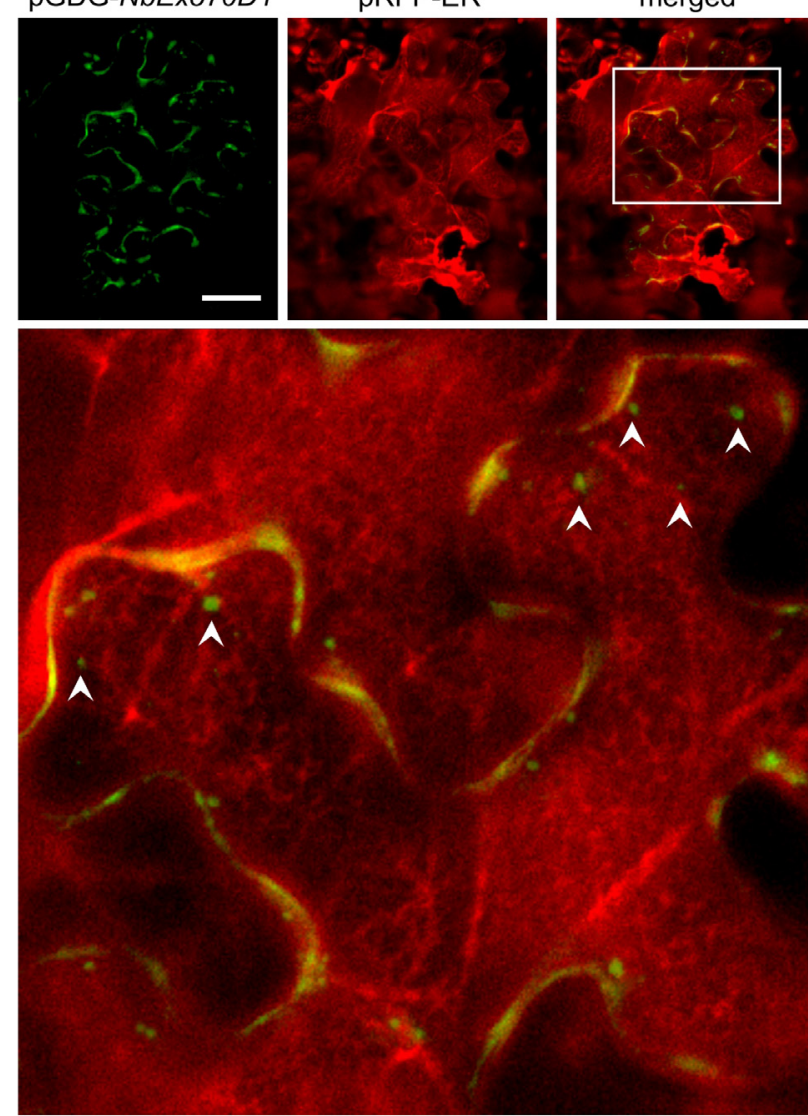

Fig. 4. The N-terminal green fluorescent protein (GFP) target NbExo70D1 co-expressed with red fluorescent proteinendoplasmic reticulum in Nicotiana benthamiana leaf epidermal cells. The square in "merged" was magnified. Arrowheads indicate the localization of GFP-NbExo70D1 in punctate to endoplasmic reticulum White bars - $10 \mu \mathrm{m}$.

nbexo70d1 domain $D$ mutant seedlings were treated with $\mathrm{NaCl}$ or $\mathrm{KCl}$. The growth of seedlings was monitored by measuring root length after $5 \mathrm{~d}$ of growth (Fig. 5 Suppl.). In the absence of salt treatments (i.e., in the negative control), the roots of the wild type and mutant plants grew at the same rate. At $100 \mathrm{mM} \mathrm{NaCl}$, root growth was slower for both the wild type and the mutant plants, but the root growth of the mutants was faster than of the wild type seedlings; the mutants had longer primary roots. At $200 \mathrm{mM} \mathrm{NaCl}$, the growth of roots in the mutant plants was strongly inhibited; however, there was practically no growth in the wild type plants at this concentration. At $300 \mathrm{mM} \mathrm{NaCl}$, no growth was observed for both the wild type and the mutant plants. The root growth of the wild type and the mutant seedlings showed inhibition in response to $\mathrm{KCl}$ treatment, similar to that of the $\mathrm{NaCl}$ treatment (Fig. 6 Suppl.).

In order to determine whether the increase in salt tolerance conferred by the overexpression of dominant negative nbexo70d1 domain $D$ mutations is also effective at the vegetative growth stage, leaf discs were treated with various concentrations of $\mathrm{NaCl}$ (ranging from $0 \mathrm{mM}$ to $300 \mathrm{mM}$ ) for $4 \mathrm{~d}$. There was comparatively less bleaching in the mutant plants relative to the wild type plants under $\mathrm{NaCl}$ treatment (Fig. 5 Suppl.). The leaf discs from the wild type showed more yellowing when supplemented with $100 \mathrm{mM} \mathrm{NaCl}$ than the leaf discs from the control. Conversely, the leaf discs from the mutant plants remained green. After incubation in the higher concentrations of $\mathrm{NaCl}$ (200 and $300 \mathrm{mM}$ ), signs of bleaching or chlorosis appeared in the leaf discs from both the mutants and the wild type plants. However, $\mathrm{NaCl}$ treatment led to a more severe damage in the leaf discs of wild type plants. Mutant plants had also a higher chlorophyll content than the wild type plants (Fig. 5 Suppl.).

Exposure to high salinity imposes both osmotic and ionic stresses to plants. To distinguish them, the wild type and nbexo70d1 domain $D$ mutation seedlings were subjected to an iso-osmotic medium created by $\mathrm{NaCl}$ and nonionic osmolyte sorbitol. The root growth was inhibited also by sorbitol, but there were no differences in the effects of sorbitol between the wild type and the nbexo70d1 domain $D$ mutants after $5 \mathrm{~d}$. These findings indicate that the changes in response to salt stress in the dominant negative nbexo70d1 domain $D$ mutant plants in comparison to wild-type were rather in response to ionic than to osmotic stress.

Specific subcellular localization patterns have been observed for the exocyst protein complex (Exo70p) during processes of polarized or regulated secretion in a yeast system (Boyd et al. 2004). Also in A. thaliana, AtExo70A1, AtExo70B2, AtExo70C2, AtExo70D2, AtExo70H1, and AtExo70H7 are mostly distributed throughout the cytosol and within the nucleus (Chong et al. 2010). In addition, Wang et al. (2010) reported that AtExo70A1, AtExo70B1, and AtExo70E2 are located in both the plasma membrane and the cytoplasm. To further examine NbExo70D1 function under normal growth conditions, we conducted a subcellular localization analysis on the NbExo70D1 protein. We fused GFP to NbExo70D1 under the control of the Cauliflower mosaic virus $35 \mathrm{~S}$ promoter (Fig. $3 A$ ). In a transient expression assay, we introduced the GFP-NbExo70D1 fusions (and an emptyvector control) into leaf epidermal cells of $N$. benthamiana through mediated infiltration by Agrobacterium. The cells were stained with FM4-64 to highlight the plasma membranes and intracellular membranes and were examined by fluorescence microscopy. Co-expressions of different combinations of $\mathrm{C}$ - and N-terminally tagged GFP-NbExo70D1 show that the protein was frequently distributed in the plasma membrane and cytoplasm. Conversely, the GFP signal for the empty vector control was distributed throughout the epidermal cells (Fig. 3B). When the leaf epidermal cells were treated with $10 \mu \mathrm{M}$ FM4-64, intracellular membranes labelled with the dye FM4-64 co-localized with the GFP-NbExo70D1 protein. The accumulation of protein fusions was validated by Western blot analyses of total protein extracts from agroinfiltrated leaves (Fig. 7 Suppl.). Co-expression with red fluorescentprotein-endoplasmic reticulum indicated the localization of GFP-NbExo70D1 in the ER (Fig. 4). Thus, these results suggest that NbExo70D1 is a cytosol protein.

We further examined the localization of nbexo70d1 
A pSITEII-4C1
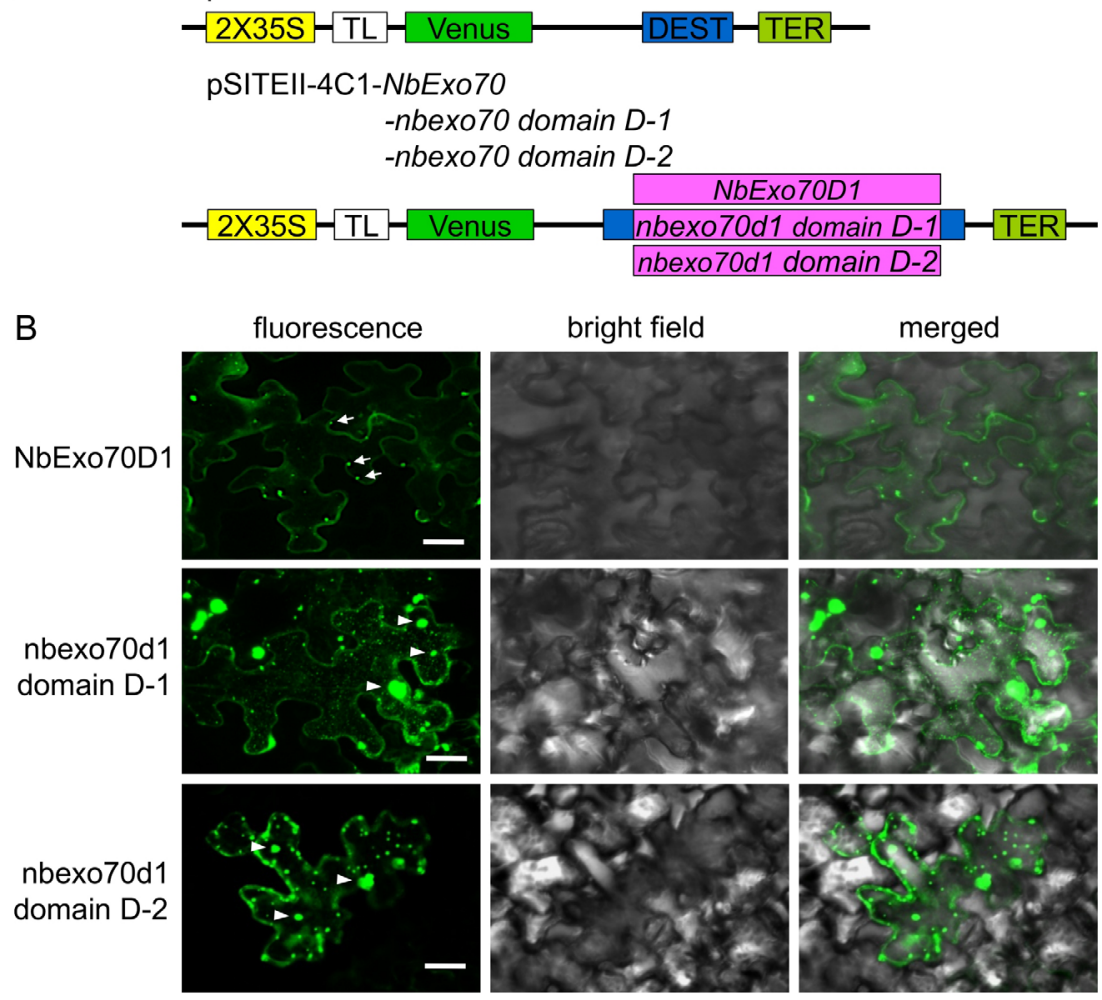

C

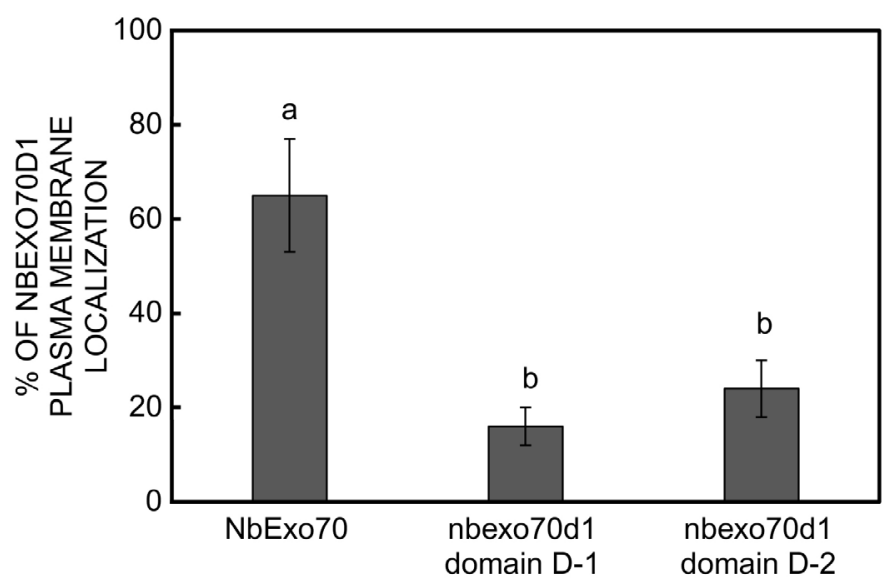

Fig. 5. Localization of $N b E x o 70 D 1$ and nbexo70d1 domain $D$ mutations in Nicotiana benthamiana epidermal cells. $A$ - A schematic diagram representing constructs used for transient transformation of $N$. benthamiana leaf epidermal cells (2X35S - tandem CaMV 35S promoter, TEV - tobacco etch virus, TL - TEV leader, DEST - destination cassette, TER - CaMV 35S transcriptional terminator). $B$ - Localization of YFP:NbExo70D1 and YFP:nbexo70d1 domain D mutations in the epidermal cells of N. benthamiana. Merged, and merging of yellow fluorescent protein (YFP) and bright field images; fluorescence, representing the YFP signals. Arrowheads indicate the aggregated Venus fusion of the NbExo70D1 protein (white bars $-10 \mu \mathrm{m}$ ). C - Percentage of NbExo70D1 localization in the plasma membrane of the wild type and transgenic $N$. benthamiana plants. The different letters indicate statistically significant differences (ANOVA with the Tukey post-hoc test; $P<0.05$ ).

domain D proteins in the epidermal cells of the mutant plants carrying YFP-nbexo70d1 domain D constructs by a confocal microscope (Fig. 5A). The YFP-nbexo70d1 domain $\mathrm{D}$ mutant proteins formed aggregates (Fig. $5 B$ ) and were mostly localized in the cytoplasm without localization in the plasma membrane (Fig. 5C). These results suggest that binding NbExo70D1 with the plasma membrane was disrupted in the cell of the nbexo70d1 domain $D$ mutants.
To investigate the association between NbExo70D1 and autophagy, we generated transformants with fused GFP-NbExo70D1 and the autophagic marker NbATG8fRFP. In addition, we used a ROS inducer MV to trigger autophagy in $N$. benthamiana (Xiong et al. 2007). Nitro blue tetrazolium and DAB staining indicate that MV was positively correlated with the accumulation of superoxide anion and $\mathrm{H}_{2} \mathrm{O}_{2}$, and NbATG8f-RFP did not co-localized 
with GFP-NbExo70D1 in the cytoplasm indicating that NbExo70D1was not related to autophagy (Fig. 8 Suppl.).

In order to investigate the indirect effect of salt stress on the nbexo70d1 domain $D$ mutant plants, ROS production in the roots of the wild type and mutant seedlings during salt treatment were tested and compared. The accumulations of superoxide anion and $\mathrm{H}_{2} \mathrm{O}_{2}$ were also determined by staining using NBT and DAB, respectively. A similar pattern of ROS accumulation was observed using this method in the shoots and roots of the control wild type and mutant seedlings (Fig. 9 Suppl.). A high amount of ROS were observed in the roots of the wild type seedlings, but not in the mutant roots after $24 \mathrm{~h}$ exposure to $200 \mathrm{mM}$ $\mathrm{NaCl}$ (Fig. 9 Suppl.). The generation and accumulation of ROS were observed already several minutes after initiation of salt treatment in both the wild type and the mutant plants. Staining ROS by $\mathrm{CM}-\mathrm{H}_{2}$ DCFDA shows that ROS were generated and distributed in the root cell tonoplast of the wild type plants. Conversely, the stained ROS only appeared as spots in the root cells in the mutant plants (Fig. 9 Suppl.). Moreover, salt stress-induced ROS generation was inhibited after the addition of diphenylene iodonium (DPI), an NADPH oxidase inhibitor. The addition was done both during the initiation of salt treatment and $30 \mathrm{~min}$ before the observation.

To determine whether NADPH oxidase was directly involved in reduction of salt stress-induced ROS production in the nbexo $70 d 1$ domain $D$ mutant plants, NADPH oxidase activity in the wild type and nbexo70d1 domain $D$ mutant plants under salt treatment were characterized using an in-gel and XTT assays. The results show that the activity of NADPH oxidase in trhe wild type tobacco plants was induced after treatment with $200 \mathrm{mM} \mathrm{NaCl}$ for $30 \mathrm{~min}$ and was inhibited by DPI (Fig. 9 Suppl. and Fig. 10 Suppl.). In contrast, the NADPH oxidase activity of the nbexo70d1 domain $D$ mutant plants was lower than of the wild type plants and did not change under $\mathrm{NaCl}$ treatment regardless whether DPI was present or not (Fig. 9 Suppl. and Fig. 10 Suppl.). These results suggest that the dominant negative mutant of nbexo70d1 domain $D$ may affect NADPH oxidase activity under salt treatment.

The cell can regulate outcome of stress through adjusting the rate of vesicle trafficking, including exocytosis and endocytosis at the plasma membrane (Levine 2002). This may be a result of membrane recycling or improved delivery of essential cargo to its destination, or of trafficassociated signal transduction (Levine et al. 2001, Surpin and Raikhel 2004, Sutter et al. 2006). In plants, the octameric exocyst complex, including the extremely large family of Exo70 paralogs, is implicated in tethering post-Golgi secretory vesicles to the plasma membrane (Li et al. 2010). Increased expression of AtExo70 genes was found in response to salt stress (Chong et al. 2010, Julkowska et al. 2016). In the present study, the activity of $\mathrm{NbExo} 70 \mathrm{D} 1$ promoter in roots, leaves, and reproductive organs was strongly induced by salt treatment (Fig. $2 A, B$ ). These results reveal the potential involvement of Exo70 genes in plant response to salt stress.

The association of the exocyst with the plasma membrane is a crucial step in vesicle tethering (Cai et al.
2007, He et al. 2007). Exo70 is a central component of the mammalian exocyst complex, direct binding to $\mathrm{e}[\operatorname{PtdIns}(4,5) \mathrm{P} 2]$ in the plasma membrane for exocytosis (He et al. 2007). In addition, Exo70 participates in the regulation of actin dynamics by interaction with the Arp2/3 nucleation complex via a motif almost overlapping with the PtdIns(4,5)P2-binding site (Zuo et al. 2006). Manipulating the PtdIns(4,5)P2 distribution affected exocytosis via its effect on actin organization (Roth 2004). Binding Exo70 to PtdIns(4,5)P2 was confined to a motif at the C-terminus of a rod shaped molecule, conserved in the Exo70 of yeast, D. melanogaster, mammals, rice, and Arabidopsis (Fig. 2 Suppl.). A subset of the positively charged residues at the C-terminus of Exo70 is essential for this interaction (He et al. 2007). It was found that the interaction between Arabidopsis Sec3 and Exo70A1 subunits (Hála et al. 2008), which, together with the likely ability of Exo70A1 (and some other Exo70 paralogues) to bind membrane phospholipids, suggests that plant Exo70s might serve as landmarks for exocyst vesicle targeting (Žárský et al. 2009). Hutagalung et al. (2009) demonstrated that the domain D of Exo70p in yeast is essential and that exo70 $\triangle d D$ is a recessive loss-of-function mutation. Our results (Fig. 4 Suppl.) are consistent with the finding of an earlier study in yeast cells, where mutations in the C-terminus of Exo70 (that disrupt its association with phospholipids) have no effects on growth (He et al. 2007). Previous studies showed that Exo70 and another exocyst component, Sec3, may function in tandem to mediate the anchorage of the exocyst complex to plasma membrane (Guo et al. 2001, He et al. 2007). Disrupting the Exo70phospholipid interaction alone is not sufficient to abolish the plasma membrane targeting of the exocyst, which may explain the apparent lack of negative effects on growth in the nbexo70d1 domain $D$ mutants. Using a ROS inducer MV, Xiong et al. (2007) showed that ROS can induce autophagy in A. thaliana. Furthermore, co-localization of an autophagy marker AtATG8f and Exo70B1 in both the cytoplasm and the vacuole suggests there is a relationship between Exo70 and autophagy (Kulich et al. 2013). However, our results do not support the hypothesis that there is a relationship between NbExo70D1 and ROSinduced autophagy (Fig. 8 Suppl.).

Overproduction of ROS has been observed in plants exposed to a number of stresses, and it is considered as a factor causing oxidative stress (Bowler et al. 1992). A process of endogenous production of ROS in the intracellular communication system that regulates stress response was proposed in Fedoroff (2006). A low content of ROS act as a signal for activation of stress response pathways, e.g., activate the expression of stress-responsive genes for producing transcriptional factors, protein kinases, enzymes, molecular chaperones, and other functional proteins (Baxter et al. 2013, You and Chan 2015). Reactive oxygen species produced by NADPH oxidase was indicated as crucial players in plant stress responses (Yoshioka et al. 2003). In addition, the traffic destination of ROS under salt stress is regulated by vesicle trafficking complexes (Sanderfoot et al. 2000, Leshem et al. 2006). Along with the findings of previous studies 
(Mazel et al. 2004, Leshem et al. 2007, Banu et al. 2009), we have shown that a high accumulation of ROS can be observed under salt stress (Fig. 9 Suppl.).

Exo70 participates in anchoring and fusing secretory and transport vesicles to the target plasma membrane, but Exo70 was demonstrated also to play regulatory roles in processes not involved in membrane trafficking in plants (Sekereš et al. 2017). Disrupting membrane targeting NbExo70D1 in the nbexo70d1 domain $D$ mutant seedlings resulted in a lower ROS production under salt stress in the shoots and roots as compared with the wild type seedlings (Fig. 9 Suppl.). Reactive oxygen species accumulation under salt stress markedly decreased in the roots treated with brefeldin A, a general vesicle trafficking inhibitor, thus, the inhibition of membrane trafficking can inhibit ROS accumulation (Leshem et al. 2007, Lin et al. 2013). Potocký et al. (2012) determined that NADPH oxidase activity is modulated by calcium ions, signaling phospholipids and Rac/Rop GTPases. Reactive oxygen species production under salt stress can be caused by a defective electron flow due to altered membrane ionic interactions, leading to electron leakage toward oxygen (Borsani et al. 2001). Moreover, vesicle trafficking leads to the intracellular activation of NADPH oxidase, which generates ROS that act in the signaling the salt responses (Mazel et al. 2004, Leshem et al. 2006). In this study, we found that two Nbrboh genes (NbrbohA and NbrbohB) were up regulated in the tobacco seedlings during salt stress (Fig. 11 Suppl.). In addition, using DPI, an NADPH oxidase inhibitor, we show that $\mathrm{NaCl}$ triggered ROS generation in the wild type and mutant tobacco roots was inhibited (Fig. Suppl.). Furthermore, the results from ingel and XTT assays demonstrate that NADPH oxidase activity was lower in the nbexo70d1 domain $D$ mutants as compared with the wild type suggesting that NADPH oxidase is directly involved in ROS production under salt stress (Fig. 9 Suppl. and Fig. 10 Suppl.). Overexpression of the dominant negative nbexo70d1 domain $D$ in mutants enhanced tolerance against the high salinity via the disruption of Exo70 binding to the plasma membrane (Fig. 5 Suppl.).

In conclusion, our results reveal that in plants under salt stress, the interaction of Exo70 with the plasma membrane may play a key role in the regulation of ROS production by NADPH oxidases in the plasma membrane. As a result of a dominant negative effect on ROS generation and the oxidative burst under salt stress, the disruption of membrane targeting NbExo70D1 by the mutations in NbExo70D1 domain $D$ increased salt stress tolerance in plants.

\section{References}

An, Y., Ji, J., Wu, W., Lv, A., Huang, R., Wei, Y.: A rapid and efficient method for multiple-site mutagenesis with a modified overlap extension PCR. - Appl. Microbiol. Biotechnol. 68: 774-778, 2005.

Arnon, D.I.: Copper enzymes in isolated chloroplast. Polyphenoloxidase in Beta vulgaris. - Plant Physiol. 24: 1-15, 1949.
Banu, M.N.A., Hoque, M.A., Watanabe-Sugimoto, M., Matsuoka, K., Nakamura, Y., Shimoishi, Y., Murata, Y.: Proline and glycinebetaine induce antioxidant defense gene expression and suppress cell death in cultured tobacco cells under salt stress. - J. Plant Physiol. 166: 146-156, 2009.

Baxter, A., Mittler, R., Suzuki, N.: ROS as key players in plant stress signalling. - J. exp. Bot. 65: 1229-1240, 2013.

Bhaskar, P.B., Venkateshwaran, M., Wu, L., Ané, J.M., Jiang, J.: Agrobacterium-mediated transient gene expression and silencing: a rapid tool for functional gene assay in potato. PloS ONE 4: e5812, 2009.

Borsani, O., Valpuesta, V., Botella, M.A.: Evidence for a role of salicylic acid in the oxidative damage generated by $\mathrm{NaCl}$ and osmotic stress in Arabidopsis seedlings. - Plant Physiol. 126: 1024-1030, 2001.

Bowler, C., Montagu, M.V., Inze, D.: Superoxide dismutase and stress tolerance. - Annu. Rev. Plant Physiol. Plant mol. Biol. 43: 83-116, 1992.

Boyd, C., Hughes, T., Pypaert, M., Novick, P.: Vesicles carry most exocyst subunits to exocytic sites marked by the remaining two subunits, Sec3p and Exo70p. - J. cell. Biol. 167: 889-901, 2004.

Cai, H., Reinisch, K., Ferro-Novick, S.: Coats, tethers, Rabs, and SNAREs work together to mediate the intracellular destination of a transport vesicle. - Dev. Cell 12: 671-682, 2007.

Chong, Y.T., Gidda, S.K., Sanford, C., Parkinson, J., Mullen, R.T., Goring, D.R.: Characterization of the Arabidopsis thaliana exocyst complex gene families by phylogenetic, expression profiling, and subcellular localization studies. New Phytol. 185: 401-419, 2010.

Cvrckova, F., Grunt, M., Bezvoda, R., Hala, M., Kulich, I., Rawat, A., Zarsky, V.: Evolution of the land plant exocyst complexes. - Front. Plant Sci. 3: 159-170, 2012.

Dong, G., Hutagalung, A.H., Fu, C., Novick, P., Reinisch, K.M.: The structures of exocyst subunit Exo70p and the Exo84p C-terminal domains reveal a common motif. - Nat. struct. mol. Biol. 12: 1094-1100, 2005.

Du, Y., Berg, J.A., Govers, F., Bouwmeester, K., Overdijk, E.J.R. Solanaceous exocyst subunits are involved in immunity to diverse plant pathogens. - J. exp. Bot. 69: 655-666, 2018.

Fedoroff, N.: Redox regulatory mechanisms in cellular stress responses. - Ann. Bot. 98: 289-300, 2006.

Goodin, M.M., Dietzgen, R.G., Schichnes, D., Ruzin, S., Jackson, A.O.: pGD vectors: versatile tools for the expression of green and red fluorescent protein fusions in agroinfiltrated plant leaves. - Plant J. 31: 375-383, 2002.

Guo, W., Tamanoi, F., Novick, P.: Spatial regulation of the exocyst complex by Rhol GTPase. - Nat. cell. Biol. 3: 353$360,2001$.

Hála, M., Cole, R., Synek, L., Drdová, E., Pečenková, T., Nordheim, A., Lamkemeyer, T., Madlung, J., Hochholdinger, F., Fowler, J.E., Žárský, V.: An exocyst complex functions in plant cell growth in Arabidopsis and tobacco. - Plant Cell 20: 1330-1345, 2008.

Hazuka, C.D., Foletti, D.L., Hsu, S.-C., Kee, Y., Hopf, F.W., Scheller, R.H.: The sec6/8 complex is located at neurite outgrowth and axonal synapse-assembly domains. - J. Neurosci. 19: 1324-1334, 1999.

He, B., Xi, F., Zhang, X., Zhang, J., Guo, W.: Exo70 interacts with phospholipids and mediates the targeting of the exocyst to the plasma membrane. - EMBO J. 26: 8, 2007.

Hirschi, K.D., Korenkov, V.D., Wilganowski, N.L., Wagner, G.J.: Expression of Arabidopsis CAX2 in tobacco. Altered metal accumulation and increased manganese tolerance. - Plant Physiol. 124: 125-134, 2000.

Ho, S.N., Hunt, H.D., Horton, R.M., Pullen, J.K., Pease, L.R.: 
Site-directed mutagenesis by overlap extension using the polymerase chain reaction. - Gene 77: 51-59, 1989.

Hong, D., Jeon, B.W., Kim, S.Y., Hwang, J.-U., Lee, Y.: The ROP2-RIC7 pathway negatively regulates light-induced stomatal opening by inhibiting exocyst subunit Exo70B1 in Arabidopsis. - New Phytol. 209: 624-635, 2016.

Hsu, S.C., TerBush, D., Abraham, M., Guo, W.: The exocyst complex in polarized exocytosis. - Int. Rev. Cytol. 233: 243265, 2004.

Hutagalung, A.H., Coleman, J., Pypaert, M., Novick, P.J.: An internal domain of Exo70p is required for actin-independent localization and mediates assembly of specific exocyst components. - Mol. Biol. Cell 20: 153-163, 2009.

Jefferson, R.A., Kavanagh, T.A., Bevan, M.W.: GUS fusions: beta-glucuronidase as a sensitive and versatile gene fusion marker in higher plants. - EMBO J. 6: 3901-3907, 1987.

Julkowska, M.M., Klei, K., Fokkens, L., Haring, M.A., Schranz, M.E., Testerink, C.: Natural variation in rosette size under salt stress conditions corresponds to developmental differences between Arabidopsis accessions and allelic variation in the LRR-KISS gene. - J. exp. Bot. 67: 2127-2138, 2016.

Kilian, J., Whitehead, D., Horak, J., Wanke, D., Weinl, S., Batistic, O., D'Angelo, C., Bornberg-Bauer, E., Kudla, J., Harter, K.: The AtGen express global stress expression data set: protocols, evaluation and model data analysis of UV-B light, drought and cold stress responses. - Plant J. 50: 347363, 2007.

Kulich, I., Pečenková, T., Sekereš, J., Smetana, O., Fendrych, M., Foissner, I., Höftberger, M., Žárský, V.: Arabidopsis exocyst subcomplex containing subunit EXO70B1 is involved in autophagy-related transport to the vacuole. - Traffic 14: 11551165,2013

Leshem, Y., Melamed-Book, N., Cagnac, O., Ronen, G., Nishri, Y., Solomon, M., Cohen, G., Levine, A.: Suppression of Arabidopsis vesicle-SNARE expression inhibited fusion of $\mathrm{H}_{2} \mathrm{O}_{2}$-containing vesicles with tonoplast and increased salt tolerance. - Proc. nat. Acad. Sci. USA 103: 18008-18013, 2006.

Leshem, Y., Seri, L., Levine, A.: Induction of phosphatidylinositol 3-kinase-mediated endocytosis by salt stress leads to intracellular production of reactive oxygen species and salt tolerance. - Plant J. 51: 185-197, 2007.

Levine, A.: Regulation of stress responses by intracellular vesicle trafficking? - Plant Physiol. Biochem. 40: 531-535, 2002.

Levine, A., Belenghi, B., Damari-Weisler, H., Granot, D.: Vesicleassociated membrane protein of Arabidopsis suppresses Baxinduced apoptosis in yeast downstream of oxidative burst. - J. biol. Chem. 276: 46284-46289, 2001.

Li, C.R., Lee, R.T.H., Wang, Y.M., Zheng, X.D., Wang, Y.: Candida albicans hyphal morphogenesis occurs in Sec3pindependent and Sec3p-dependent phases separated by septin ring formation. - J. cell. Sci. 120: 1898-1907, 2007.

Li, S., Van Os, G.M.A., Ren, S., Yu, D., Ketelaar, T., Emons, A.M.C., Liu, C.-M.: Expression and functional analyses of Exo70 genes in Arabidopsis implicate their roles in regulating cell type-specific exocytosis. - Plant Physiol. 154: 1819-1830, 2010.

Lin, C.Y., Trinh, N.N., Fu, S.F., Hsiung, Y.C., Chia, L.C., Lin, C.W., Huang, H.J.: Comparison of early transcriptome responses to copper and cadmium in rice roots. - Plant mol. Biol. 81: 507-522, 2013

Lin, Y., Ding, Y., Wang, J., Shen, J., Kung, C.H., Zhuang, X., Cui, Y., Yin, Z., Xia, Y., Lin, H., Robinson, D.G., Jiang, L.: Exocyst-positive organelles and autophagosomes are distinct organelles in plants. - Plant Physiol. 169: 1917, 2015.

Liu, J., Zuo, X., Yue, P., Guo, W.: Phosphatidylinositol 4,5-bisphosphate mediates the targeting of the exocyst to the plasma membrane for exocytosis in mammalian cells. - Mol. Biol. Cell 18: 4483-4492, 2007.

Martin, K., Kopperud, K., Chakrabarty, R., Banerjee, R., Brooks, R., Goodin, M.M.: Transient expression in Nicotiana benthamiana fluorescent marker lines provides enhanced definition of protein localization, movement and interactions in planta. - Plant J. 59: 150-162, 2009.

Mazel, A., Leshem, Y., Tiwari, B.S., Levine, A.: Induction of salt and osmotic stress tolerance by overexpression of an intracellular vesicle trafficking protein AtRab7 (AtRabG3e). - Plant Physiol. 134: 118-128, 2004.

Pérez-Pérez, M.E., Lemaire, S.D., Crespo, J.L.: Reactive oxygen species and autophagy in plants and algae. - Plant Physiol. 160: 156-164, 2012.

Pommereit, D., Wouters, F.S.: An NGF-induced Exo70-TC10 complex locally antagonises Cdc42-mediated activation of N-WASP to modulate neurite outgrowth. - J. cell. Sci. 120: 2694-2705, 2007.

Potocký, M., Pejchar, P., Gutkowska, M., Jiménez-Quesada, M.J., Potocká, A., Alché, J.d.D., Kost, B., Žárský, V.: NADPH oxidase activity in pollen tubes is affected by calcium ions, signaling phospholipids and Rac/Rop GTPases. - J. Plant Physiol. 169: 1654-1663, 2012.

Rawat, A., Brejšková, L., Hála, M., Cvrčková, F., Žárský, V.: The Physcomitrella patens exocyst subunit EXO70.3d has distinct roles in growth and development, and is essential for completion of the moss life cycle. - New Phytol. 216: 438454, 2017

Rossé, C., Hatzoglou, A., Parrini, M.C., White, M.A., Chavrier, P., Camonis, J.: RalB mobilizes the exocyst to drive cell migration. - Mol. cell. Biol. 26: 727-734, 2006.

Roth, M.G.: Phosphoinositides in constitutive membrane traffic. - Physiol. Rev. 84: 699-730, 2004.

Sagi, M., Fluhr, R.: Superoxide production by plant homologues of the gp91phox NADPH oxidase. Modulation of activity by calcium and by tobacco mosaic virus infection. - Plant Physiol. 126: 1281-1290, 2001.

Sanderfoot, A.A., Assaad, F.F., Raikhel, N.V.: The Arabidopsis genome. An abundance of soluble N-ethylmaleimidesensitive factor adaptor protein receptors. - Plant Physiol. 124: 1558-1569, 2000.

Sato, T.K., Overduin, M., Emr, S.D.: Location, location, location: membrane targeting directed by PX domains. - Science 294: 1881-1885, 2001.

Sekereš, J., Pejchar, P., Šantrůček, J., Vukašinović, N., Žárský, V., Potocký, M.: Analysis of exocyst subunit EXO70 family reveals distinct membrane polar domains in tobacco pollen tubes. - Plant Physiol. 173: 1659-1675, 2017.

Seo, D.H., Ahn, M.Y., Park, K.Y., Kim, E.Y., Kim, W.T.: The N-Terminal UND motif of the Arabidopsis U-Box E3 Ligase PUB18 is critical for the negative regulation of ABA-mediated stomatal movement and determines its ubiquitination specificity for exocyst subunit Exo70B1. - Plant Cell 28: 2952-2973, 2016.

Surpin, M., Raikhel, N.: Plant cell biology: traffic jams affect plant development and signal transduction. - Nat. Rev. mol. cell. Biol. 5: 100-109, 2004.

Sutter, J.U., Campanoni, P., Tyrrell, M., Blatt, M.R.: Selective mobility and sensitivity to SNAREs is exhibited by the Arabidopsis KAT1 $\mathrm{K}^{+}$channel at the plasma membrane. Plant Cell 18: 935-954, 2006.

TerBush, D.R., Maurice, T., Roth, D., Novick, P.: The exocyst is a multiprotein complex required for exocytosis in Saccharomyces cerevisiae. - EMBO J. 15: 6483-6494, 1996.

TerBush, D.R., Novick, P.: Sec6, Sec8, and Sec 15 are components 
of a multisubunit complex which localizes to small bud tips in Saccharomyces cerevisiae. - J. cell. Biol. 130: 299-312, 1995.

Thordal-Christensen, H., Zhang, Z., Wei, Y., Collinge, D.B.: Subcellular localization of $\mathrm{H}_{2} \mathrm{O}_{2}$ in plants. $\mathrm{H}_{2} \mathrm{O}_{2}$ accumulation in papillae and hypersensitive response during the barleypowdery mildew interaction. - Plant J. 11: 1187-1194, 1997.

Trinh, N.-N., Huang, T.-L., Chi, W.-C., Fu, S.-F., Chen, C.-C., Huang, H.-J.: Chromium stress response effect on signal transduction and expression of signaling genes in rice. Physiol. Plant. 150: 205-224, 2014.

Trinh, N.N., Le, H.T., Dam, S.M., Nguyen, V.T.: Overexpression of NbWRKY79 enhances salt stress tolerance in Nicotiana benthamiana. - Acta Physiol. Plant. 39: 121, 2017.

Wang, J., Ding, Y., Wang, J., Hillmer, S., Miao, Y., Lo, S.W., Wang, X., Robinson, D.G., Jiang, L.: EXPO, an exocystpositive organelle distinct from multivesicular endosomes and autophagosomes, mediates cytosol to cell wall exocytosis in Arabidopsis and tobacco cells. - Plant Cell 22: 4009-4030, 2010.

Wong, H.L., Pinontoan, R., Hayashi, K., Tabata, R., Yaeno, T., Hasegawa, K., Kojima, C., Yoshioka, H., Iba, K., Kawasaki, T., Shimamoto, K.: Regulation of rice NADPH oxidase by binding of Rac GTPase to its N-terminal extension. - Plant Cell 19: 4022-4034, 2007.

Wu, H., Rossi, G., Brennwald, P.: The ghost in the machine: small GTPases as spatial regulators of exocytosis. - Trends Cell Biol. 18: 397-404, 2008.
Zuo, X., Zhang, J., Zhang Y., Hsu, S.C., Zhou, D., Guo, W.: Exo70 interacts with the Arp2/3 complex and regulates cell migration. - Nat. cell. Biol. 8: 1383-1388, 2006.

Xiong, Y., Contento, A.L., Nguyen, P.Q., Bassham, D.C.: Degradation of oxidized proteins by autophagy during oxidative stress in Arabidopsis. - Plant Physiol. 143: 291-299, 2007.

Yao, H.Y., Xue, H.W.: Signals and mechanisms affecting vesicular trafficking during root growth. - Curr. Opin. Plant Biol. 14: 571-579, 2011.

Yoshioka, H., Numata, N., Nakajima, K., Katou, S., Kawakita, K., Rowland, O., Jones, J.D.G., Doke, N.: Nicotiana benthamiana gp91phox homologs NbrbohA and NbrbohB participate in $\mathrm{H}_{2} \mathrm{O}_{2}$ accumulation and resistance to Phytophthora infestans. - Plant Cell 15: 706-718, 2003.

You, J., Chan, Z.: ROS regulation during abiotic stress responses in crop plants. - Front. Plant Sci. 6: 1092-1106, 2015.

Žárský, V., Cvrčková, F., Potocký, M., Hála, M.: Exocytosis and cell polarity in plants - exocyst and recycling domains. - New Phytol. 183: 255-272, 2009.

Zhao, J., Zhang, X., Wan, W., Zhang, H., Liu, J., Li, M., Wang, H., Xiao, J., Wang, X.: Identification and characterization of the EXO70 gene family in polyploid wheat and related species. - Int. J. mol. Sci. 20: 60, 2018.

Zuo, X., Zhang, J., Zhang, Y., Hsu, S.C., Zhou, D., Guo, W.: Exo70 interacts with the Arp2/3 complex and regulates cell migration. - Nat. cell. Biol. 8: 1383-1388, 2006. 